\title{
BMJ Open Haemorheological and haemostatic alterations in coeliac disease and inflammatory bowel disease in comparison with non-coeliac, non-IBD subjects (HERMES): a case-control study protocol
}

Zsolt Szakács, ${ }^{1,2,3}$ Beáta Csiszár, ${ }^{1,4}$ Péter Kenyeres, ${ }^{1,4}$ Patrícia Sarlós, ${ }^{1,5}$ Bálint Erőss, ${ }^{3,5}$ Alizadeh Hussain,, ${ }^{1,6}$ Ágnes Nagy, ${ }^{6}$ Balázs Kőszegi, ${ }^{7}$ Ibolya Veczák, ${ }^{5}$ Nelli Farkas, ${ }^{8}$ Emőke Bódis, ${ }^{3}$ Katalin Márta, ${ }^{1,3}$ Andrea Szentesi, ${ }^{3}$ Margit Tökés-Füzesi, ${ }^{9}$ Tímea Berki, ${ }^{10}$ Áron Vincze, ${ }^{1,2,5}$ Kálmán Tóth, ${ }^{1,4}$ Péter Hegyi, ${ }^{\oplus 1,2,3}$ Judit Bajor $^{2,5}$

To cite: Szakács Z, Csiszár B, Kenyeres P, et al. Haemorheological and haemostatic alterations in coeliac disease and inflammatory bowel disease in comparison with noncoeliac, non-IBD subjects (HERMES): a case-control study protocol. BMJ Open 2019;9:e026315. doi:10.1136/ bmjopen-2018-026315

- Prepublication history and additional material for this paper are available online. To view these files, please visit the journal online (http://dx.doi org/10.1136/bmjopen-2018026315).

$\mathrm{PH}$ and JB contributed equally.

Received 29 August 2018 Revised 18 December 2018 Accepted 11 February 2019

Check for updates

(c) Author(s) (or their employer(s)) 2019. Re-use permitted under CC BY-NC. No commercial re-use. See rights and permissions. Published by BMJ.

For numbered affiliations see end of article.

Correspondence to Dr Péter Hegyi; p.hegyi@tm-centre.org

\section{ABSTRACT}

Introduction Haemorheological and haemostatic changes predispose to the development of arterial and venous thrombotic events; however, limited information is available on the status of these changes in coeliac disease (CeD) and inflammatory bowel disease (IBD). In this study, we aim to describe the haemorheological and haemostatic profiles of CeD and IBD patients in a Hungarian cohort of patients to investigate whether any alterations contribute to elevated thrombotic risk.

Methods and analysis This is a case-control study involving newly diagnosed and followed $\mathrm{CeD}$ and IBD patients with age-matched and sex-matched non-CeD, non-IBD subjects with an allocation ratio of 1:1:1. After informed consent is obtained, a detailed medical history will be collected, including venous and arterial thrombotic risk factors and medications. Symptoms in CeD patients will be assessed with the Gastrointestinal Symptoms Rating Scale, and disease activity in IBD patients will be determined by disease-specific scores. Dietary adherence will be assessed among $\mathrm{CeD}$ patients with a thorough interview together with a measurement of self-reported adherence, dietary knowledge and urine analysis (detection of gluten immunogenic peptides). In addition to routine laboratory parameters, haemorheological (ie erythrocyte deformability and aggregation, viscosity of whole blood and plasma) and haemostatic parameters (eg, protein C, protein S and antithrombin) with immunological indicators (ie, coeliac-specific serology and antiphospholipid antibodies) will be measured from venous blood for every participant. Primary and secondary outcomes will be haemorheological and haemostatic parameters, respectively. Univariate and multivariate statistics will be used to compare $\mathrm{CeD}$ and IBD patients to control subjects. Subgroup analysis will be performed by disease type in IBD, (Crohn's disease and ulcerose colitis), dietary adherence in $\mathrm{CeD}$, and disease activity in IBD and CeD.

\section{Strengths and limitations of this study}

- Immune-mediated bowel diseases are associated with an increased risk of arterial and venous thrombosis, but specific haemorheological and haemostatic alterations are understudied in coeliac disease $(\mathrm{CeD})$ and incomplete in inflammatory bowel disease (IBD).

- This case-control study prospectively recruits newly diagnosed and followed-up cases of $\mathrm{CeD}$ and IBD with age-matched and sex-matched controls (the allocation ratio will be $1: 1: 1$, respectively) to investigate clinical and laboratory alterations predisposing to thrombosis.

- Laboratory tests include the measurement of haemorheological (ie, erythrocyte aggregation and deformability, plasma and whole blood viscosity), haemostatic parameters (eg, levels of fibrinogen, prothrombin time, protein C, protein S and antithrombin) and immunological indicators (eg, coeliac-specific serology and antiphospholipid antibodies).

- Patients will be divided by disease activity into active and inactive.

- Results should be interpreted with caution due to the single-centre nature and case-control design of the study.

Ethics and dissemination The study was approved by the Regional and Local Research Ethics Committee, University of Pécs (Ref. No. 6917). Findings will be disseminated at research conferences and in peer-reviewed journals. Trial registration number ISRCTN49677481.

\section{INTRODUCTION}

Immune-mediated disorders may affect $5 \%-7 \%$ of the population. ${ }^{1}$ These disorders 
frequently share pathways in pathogenesis as well as organ manifestations. Coeliac disease (CeD) and inflammatory bowel disease (IBD) are systemic disorders, primarily affecting the intestines. ${ }^{2}$ They impose a significant burden of complications and concomitant diseases on patients during the disease course.

$\mathrm{CeD}$ is a chronic, immune-mediated disorder, which develops on gluten ingestion in genetically susceptible individuals. ${ }^{3}$ Global prevalence of $\mathrm{CeD}$ is around $1 \%$ with geographical differences ranging from $0.14 \%$ up to $5.7 \% .^{3}$ The clinical presentation can be divided into classic, non-classic and asymptomatic forms. ${ }^{4}$ Diagnosing asymptomatic and atypical cases is challenging but important, because the disease course of these cases may be alike. ${ }^{5}$

IBD_clinically classified as Crohn's disease or ulcerative colitis-is a chronic, relapsing disorder, which develops as a result of the interaction between environmental and genetic factors, leading to immunological responses and inflammation in the gastrointestinal tract. ${ }^{6}$ IBD is a less frequent entity than CeD: the increasing prevalence of ulcerative colitis and Crohn's disease may reach $0.5 \%$ and $0.3 \%$ in Europe, respectively. ${ }^{78}$

Immune-mediated disorders may be associated with haemorheological $^{9-11}$ and haemostatic changes, ${ }^{12-14}$ thereby contributing to an increased risk of thrombotic events. ${ }^{15}$ This increased risk is manifested in $\mathrm{CeD}^{16}$ and IBD. ${ }^{17}$ Mechanisms of thrombophilia in immune-mediated disorders are complex, and acquired factors seem important. ${ }^{18}$ An altered haemorheological profile as well as the altered levels or function of pro-coagulant and anticoagulant proteins, altered activity of clotting factors contribute to the development of arterial and venous thrombotic events. ${ }^{19-23}$

Clinical presentation of CeD-associated hypercoagulability includes a wide variety of thrombosis at venous sites, pulmonary embolism, atheroembolism (stroke) and obstetric complications. ${ }^{24} 25$ A single retrospective publication examined haemostatic alterations in a small cohort of patients: sporadic cases of protein $\mathrm{C}$ and protein $\mathrm{S}$ deficiency (due to vitamin $\mathrm{K}$ malabsorption), hyperhomocysteinaemia, and antiphospholipid antibodies were identified. ${ }^{26}$ No studies have assessed the haemorheological changes in $\mathrm{CeD}$. The multifactorial aetiology of thrombosis may embrace the interplay of malabsorption (vitamin and mineral deficiencies, eg, vitamin $\mathrm{B}_{12}$ and $\mathrm{K}$ deficiency), thrombophilic autoantibodies [anti-tissue transglutaminase (tTG) and antiphospholipid antibodies], hyperhomocysteinaemia, endothelial dysfunction, accelerated atherosclerosis, thrombocytosis and thrombocyte dysfunction, hyperviscosity, and genetics. ${ }^{24}$ 26-31 Immune-mediated comorbidities ('autoimmune traits'), such as antiphospholipid syndrome, may contribute to the elevated thrombotic risk as well. ${ }^{29}$ In addition, ingestion of trace amounts of gluten may maintain a continuous pro-inflammatory response. ${ }^{32}$

IBD is associated with venous thrombosis and pulmonary embolism as well as with the cardiovascular consequences of atherosclerosis, ie, stroke and myocardial infarction. ${ }^{33}{ }^{34}$ A significant decline in anticoagulant mechanism is well-established and there are sporadic reports on activity-dependent prothrombotic haemorheological changes. ${ }^{35-38}$ However, while individual studies have focused on single outcomes of laboratory parameters, none of them have assessed the complete haemorheological profile of patients. ${ }^{39-42}$ Other risk factors include immobilisation, surgical interventions, glucocorticoid therapy, vitamin deficiencies, hyperhomocysteinaemia and chronic inflammation alone or in conjunction with the factors above. ${ }^{434}$ In the case of IBD, disease activity may be a crucial determinant of thrombotic risk. ${ }^{34}$

\section{Scope and objectives}

No studies have assessed haemorheological and haemostatic parameters within a study to provide an overall view of thrombotic risk. Since our knowledge of haemorheological and haemostatic changes is limited in $\mathrm{CeD}$ and IBD, this study aims to carry out a comprehensive evaluation of venous and arterial prothrombotic alterations in these pro-inflammatory diseases in a Hungarian cohort of patients.

1. Primary objective.

- To identify a link between prothrombotic haemorheological and haemostatic alterations and two common immune-mediated diseases $(\mathrm{CeD}$ and IBD).

2. Secondary objective.

- To investigate the effect of disease activity on the haemorheological and haemostatic profiles of $\mathrm{CeD}$ and IBD patients.

- To find an association between the dietary adherence of $\mathrm{CeD}$ patients and the haemorheological and haemostatic alterations.

- To assess the modifying effect of immunosuppressant drugs in IBD on the haemorheological and haemostatic profiles.

\section{METHODS AND ANALYSIS \\ Design}

This is a case-control study with prospective recruitment of $\mathrm{CeD}$ and IBD patients with non-CeD, non-IBD control subjects. The study does not change the routine management of subjects included (for the WHO checklist, see table 1). The study protocol was planned in accordance with the Standard Protocol Items: Recommendations for Interventional Trials 2013 Statement. ${ }^{45}$

\section{Trial organisation and steering committee}

The Centre for Translational Medicine at the University of Pécs, which was established to advance medical research in gastroenterology, is the co-ordinator and designer of the HERMES study. The centre is experienced in running investigator-initiated clinical trials. ${ }^{46}$ A steering committee will be set up to supervise the entire study process. The principal investigator (JB) and the Trial Coordinator (ZS) are responsible for organising patient recruitment, 
Table 1 WHO checklist

\section{Data category}

Primary registry and trial identifying number

Date of registration in primary registry

Secondary identifying numbers

Source(s) of monetary or material support

\section{Information}

ISRCTN49677481

05/03/2018

\section{None}

University of Pécs Medical School; Momentum Grant from the Hungarian Academy of Sciences (LP2014-10/2014); Highly Cited Publication Grant (KH 125678) from the National Research Development and Innovation Office; GINOP 2.3.2-15-2016-00048 Stay Alive, EFOP 3.6.2-16-2017-00006 Live Longer, and EFOP-3.6.3-VEKOP-16-2017-00009; Translational Medicine Foundation; and New National Excellence Programme, Ministry of Human Capacities (ÚNKP17-3-II, ÚNKP-18-3-I)

$\begin{array}{ll}\text { Primary sponsor } & \text { None } \\ \text { Secondary sponsor(s) } & \text { None }\end{array}$

Contact for public queries

Zsolt Szakács, MD, szakacs.zsolt@pte.hu

Contact for scientific queries Judit Bajor, MD, bajor.judit@pte.hu

Public title

Investigation of haemorheological and haemostatic alterations in coeliac disease and inflammatory bowel disease in comparison with healthy subjects: A casecontrol study (HERMES)

Scientific title

Haemorheological and haemostatic alterations in coeliac disease and inflammatory bowel disease in comparison with non-coeliac, non-IBD subjects: A case-control study (HERMES)

Countries of recruitment Hungary

Health condition(s) or problem(s) studied Coeliac disease and inflammatory bowel disease

Intervention(s)

Questionnaires (thrombophilia, dietary adherence, disease activity), urine collection (dietary adherence-urine-gluten immunogenic peptide detection), blood collection (haemorheological, haemostatic and immunological tests complemented with routine laboratory panel)

Key inclusion and exclusion criteria

Inclusion criteria: adult patients ( $\geq 18$ years of age) suffering from biopsyconfirmed newly diagnosed or treated coeliac disease (by ESPHGAN, ACG, WGO guidelines), or from inflammatory bowel disease (by ECCO guidelines), and non-coeliac, non-IBD subjects

Exclusion criteria: chronic diseases (chronic kidney diseases, liver cirrhosis, heart failure, active malignant diseases), acute diseases within 2 weeks of inclusion, pregnancy, thrombotic events within 1 year, systematic lupus erythematosus, and use of oral anticoagulants or antiplatelet therapy

\begin{tabular}{ll}
\hline Study type & Observational \\
\hline Date of first enrolment & $30 / 5 / 2018$ \\
\hline Target sample size & $\begin{array}{l}\text { First phase: } 50 \text { coeliac and } 50 \text { IBD patients plus control (1-3 for each patient). } \\
\text { Second phase: target number is determined by power calculation }\end{array}$ \\
\hline Recruitment status & Ongoing \\
\hline Primary outcome(s) & Haemorheological test results \\
\hline Key secondary outcomes & Haemostatic test results
\end{tabular}

IBD, inflammatory bowel disease.

data collection, sample collection, shipping, and storage, biochemical analysis, and the publication of study results.

\section{Population and eligibility}

We will include CeD patients, IBD patients, and non-CeD, non-IBD control subjects. Eligibility criteria will be as follows:

a. Inclusion criteria (applies to all subjects).
- Blood collection must be indicated with medical conditions.

- Signed informed consent.

b. Inclusion criteria (applies to specific cohorts of patients).

- CeD patients: biopsy-confirmed newly diagnosed or followed patients (with or without adhering to a gluten-free diet) aged $\geq 18$ years; the establishment of a 
diagnosis should meet the current guidelines (European Society for Paediatric Gastroenterology Hepatology and Nutrition (ESPHGAN) and American College of Gastroenterology (ACG)) ${ }^{3}{ }^{37} 48$

- IBD patients: newly diagnosed or followed-up patients (with active or remitting disease) aged $\geq 18$ years (not following a gluten-free diet); the establishment of a diagnosis should meet the current guidelines (European Crohn's and Colitis Organisation $(\mathrm{ECCO}){ }^{4950}$

- Non-CeD, non-IBD control subjects: individuals aged $\geq 18$ years (not following a gluten-free diet) in whom $\mathrm{CeD}$ and IBD can be excluded according to the recent guidelines. ${ }^{347-50}$

c. Exclusion criteria (applies to all subjects).

- Chronic conditions.

- Estimated glomerular filtration rate calculated with the Chronic Kidney Disease Epidemiology Collaboration (CKD-EPI) formula is $<60 \mathrm{~mL}$ / $\min / 1.73 \mathrm{~m}^{2}$ (CKD3 or more severe kidney failure).

- Liver cirrhosis in Child-Pugh B-C.

- Heart failure (New York Heart Association (NYHA) III-IV).

- Active malignant diseases.

- Any acute diseases or invasive procedures within 2 weeks of recruitment (eg, systemic infection, surgery, or major trauma).

- Thrombotic events within 1 year of recruitment.

- Ongoing oral anticoagulant therapy (vitamin K antagonists) and/or antiplatelet drugs.
- Confirmed systemic lupus erythematosus.

- Pregnancy.

- Patients unable to understand the essentials of the informed consent.

\section{Flow and timing}

All subjects at our academic hospital for a planned check-up or referred to the centre for diagnostic purposes will be recruited consecutively. The place of recruitment will be the Division of Gastroenterology, First Department of Medicine, University of Pécs Medical School. This tertiary centre provides professional gastroenterological care for about 300000 inhabitants in Baranya County, Hungary.

Recruitment of the study population will be managed in two phases (see Target number of patient section), with the expected recruiting period being between May 2018 and May 2019 (covering 1 year). Table 2 shows the timeline of the study. Patients will be provided with an information sheet and must provide written consent before sampling. Informed consent will be obtained by personnel with a medical degree. Participants may withdraw from the study for any reason at any time. Consent forms and other related documents will be accessible at https://tm-centre.org.

Patients will be monitored by our professional data management team throughout the entire process of data and biological sample collection to ensure perfect adherence to protocol. Written feedback will be provided to patients on the results of the laboratory tests and dietary evaluation. If findings indicate, patients will be referred

Table 2 Schedule for the study

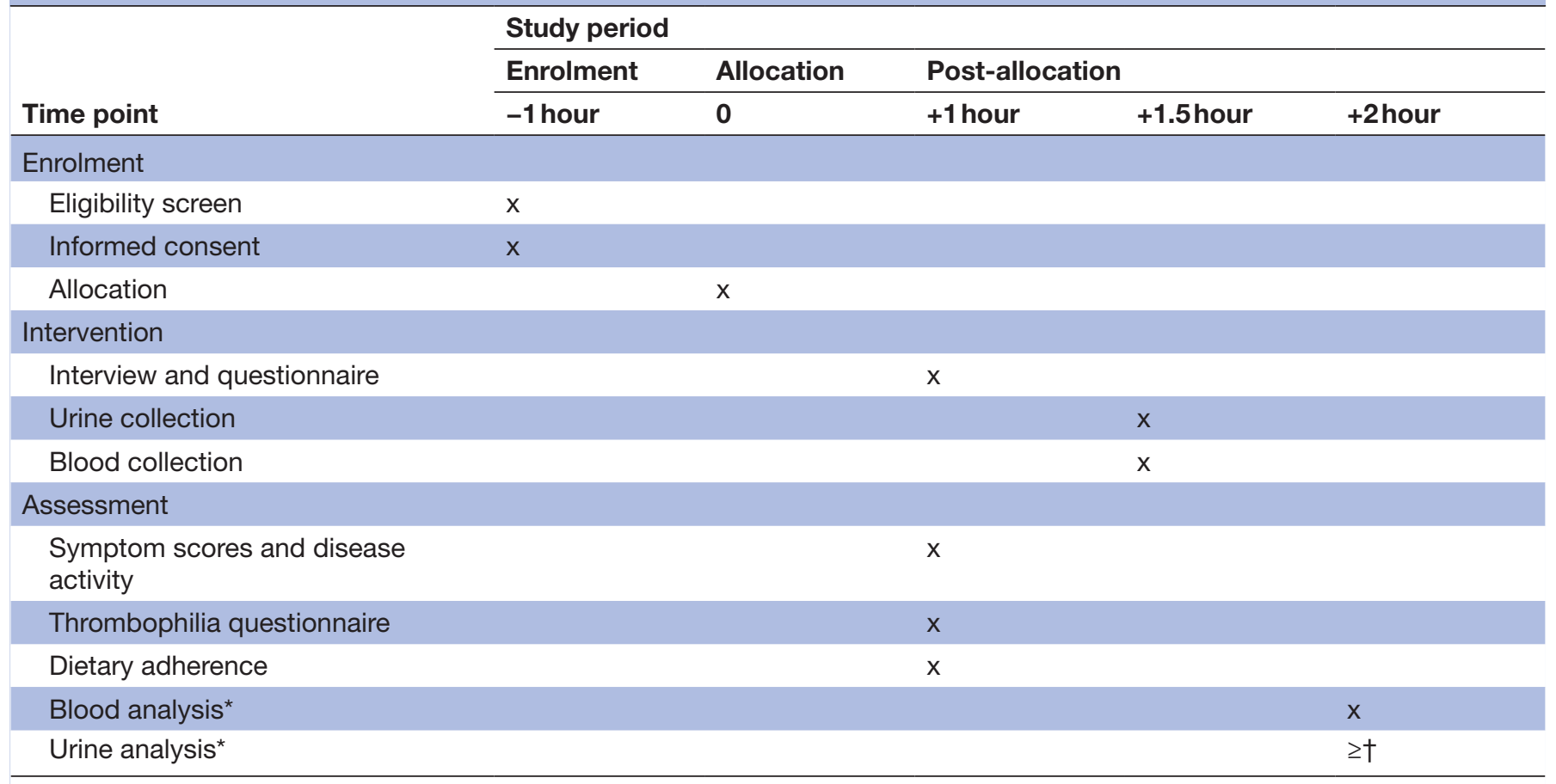

*After analysis, blood and urine residues will be stored in the biobank.

†Samples will be deep frozen until all participants have been recruited. 


\begin{tabular}{|c|c|c|c|}
\hline & $\begin{array}{l}\text { CeD } \\
\text { patients }\end{array}$ & $\begin{array}{l}\text { IBD } \\
\text { patients }\end{array}$ & $\begin{array}{l}\text { Control } \\
\text { subjects }\end{array}$ \\
\hline Thrombophilia questionnaire & + & + & + \\
\hline GSRS & + & - & + \\
\hline $\begin{array}{l}\text { Dietary interview and GFD } \\
\text { adherence tests }\end{array}$ & + & - & + \\
\hline Mayo Score/CDAI & - & + & + \\
\hline Urine GIP detection & + & - & + \\
\hline \multicolumn{4}{|l|}{ Laboratory measures } \\
\hline Routine parameters & + & + & + \\
\hline Haemorheology & + & + & + \\
\hline Haemostasis & + & + & + \\
\hline Immunological indicators & + & + & + \\
\hline
\end{tabular}

CDAl, Crohn's Disease Activity Index; CeD, coeliac disease;

GFD, gluten-free diet; GIP, gluten-immunogenic peptides; GSRS, Gastrointestinal Symptoms Rating Scale; IBD, inflammatory bowe disease.

to their general practitioners or a specialist for further investigation and management.

\section{Measurements}

All samples will be collected and questionnaires will be administered within 2 hours after allocation. Actions for each group are defined and listed in table 3 .

Detailed history (including medications for preceding 3 months) and risk factors of venous and arterial thrombotic events will be covered with a $15 \mathrm{~min}$ thrombophilia questionnaire (administered by a person with a medical degree).

The Gastrointestinal Symptoms Rating Scale is a tool designed to assess the severity of gastrointestinal symptoms on a scale of 1-7 (administered by a person with a medical degree).$^{51}$

Disease activity in IBD will be estimated with either the (modified) Mayo Score ${ }^{52}$ or Crohn's Disease Activity Index $^{53}$ in patients with ulcerative colitis and Crohn's disease, respectively, while tissue transglutaminase (tTG) levels will be used to measure the activity of CeD. (Scores will be determined by the gastroenterologist enrolling the patient.)

Dietary adherence of CeD patients will be estimated through (1) a dietary interview conducted by a trained dietitian on a scale of $1-10$, (2) self-reporting, ${ }^{54}$ (3) a test measuring knowledge of gluten-free foods, (4) urine gluten-immunogenic peptides (GIP) detection (details in the text) and (5) coeliac-specific serology [tTG and endomysium antibody levels (EMA) $].{ }^{55}$ Patients will be divided into those with good and poor dietary adherence based on the complex assessment of the above-mentioned data.

All laboratory tests will be performed in the same laboratory (University of Pécs, Hungary) from venous blood. Blood samples will be collected in plastic tubes prospectively [ 2 x BD Vacutainer $10.0 \mathrm{~mL}$ (red), 2 x BD Vacutainer
$6.0 \mathrm{~mL}$ (purple), 1 x BD Vacutainer $3.0 \mathrm{~mL}$ (pink), 1 x BD Vacutainer $2.7 \mathrm{~mL}$ (blue) and $1 \mathrm{x}$ BD Seditainer $5.0 \mathrm{~mL}$ (black) for a total of $42.7 \mathrm{~mL}$ blood from each patient (BD, USA)].

We will measure:

- Routine laboratory parameters: bilirubin, urea, creatinine, cholesterol (total, high-density and low-density lipoproteins), triglyceride, , aspartate aminotransferase, alanine aminotransferase, alkaline phosphatase, gamma-glutamyl transferase, total protein, albumin, immunoglobulins, $\mathrm{C}$ reactive protein, vitamin $\mathrm{B}_{12}$, folic acid, homocysteine, blood counts and erythrocyte sedimentation.

- Immunological indicators: antiphospholipid antibodies (lupus anticoagulant, cardiolipin IgG/IgA/ IgM, B2-glycoprotein-I IgG/IgA/IgM, prothrombin $\mathrm{IgG} / \mathrm{IgA} / \mathrm{IgM}$ ) and coeliac-specific antibodies (tTG IgA/IgG, EMA IgA).

- Haemostatic parameters: prothrombin, thrombin time, activated partial thromboplastin time, fibrinogen, antithrombin activity, protein $\mathrm{C}$ activity and protein $\mathrm{S}$ activity.

- Haemorheological parameters: erythrocyte aggregation by Myrenne aggregometer (model MA-1, Myrenne GmbH, Roetgen, Germany) and Laser-assisted Optical Rotational Cell Analyzer (LORCA, R\&R Mechatronics, Hoorn, The Netherlands); erythrocyte deformability with laser-diffraction ektacytometry with a LORCA; and viscosity of whole blood and plasma by Brookfield DV-III Ultra LV Programmable rotational viscometer (Brookfield Engineering Labs; Middleboro, Mass., USA). The Case Report Form providing data about the measurements is presented in online supplementary material.

Strict adherence will be kept during the haemorheological tests to the guidelines proposed by the International Expert Panel for Standardisation of Haemorheological Methods. ${ }^{56}$ The fact that equipment for haemorheological measurements is not available in other centres in Hungary and that blood samples must be processed within 2 hours of sampling without freezing restricted our expansion of this project to a multicentre study.

An extra tube will be collected and stored for further haemostatic measurements (eg, clotting factors) if any abnormality of parameters measured is detected.

Midstream urine (at least $100 \mathrm{~mL}$ ) will be collected in sterile urine sample containers. Samples will be stored at $4^{\circ} \mathrm{C}$ until transfer to the Biobank at the Institute for Translational Medicine, University of Pécs Medical School, on the day of sampling, where samples will be deep frozen at $-80^{\circ} \mathrm{C}$. After preparation, urine GIP detection will be performed with Biomedal (Spain) products.

\section{Outcomes}

\section{Primary.}

- Haemorheological test results (erythrocyte aggregation and deformability, whole blood and plasma viscosity). 
Table 4 Blinding of personnel included in the study

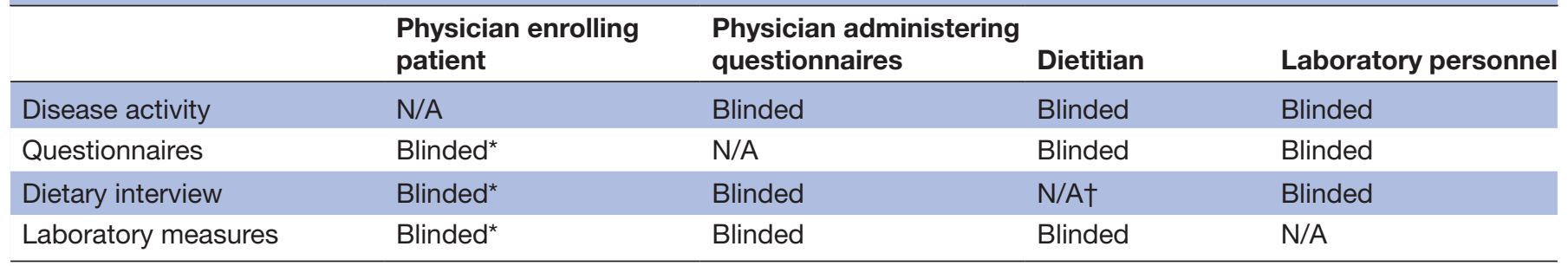

${ }^{*}$ The treating physician will immediately access data for safety reasons and act accordingly. Patients will be informed of the laboratory results in a letter.

†Dietary education will be provided based on dietary adherence.

$\mathrm{N} / \mathrm{A}$, not applicable.

2. Secondary.

- Haemostatic test results (antithrombin, protein C, protein S), folic acid, and homocysteine levels.

\section{Target number of patients}

This is a two-phase study. In the first phase, we will enrol $50 \mathrm{CeD}$ and $50 \mathrm{IBD}$ patients with 50 age- matched and sex-matched control subjects; the case-control ratio will be 1:1:1, respectively. Then, an interim analysis will be performed to calculate the power for the analyses of the outcomes. If the power exceeds $80 \%$, recruitment will be considered completed; otherwise, recruitment will continue until the desired power is reached.

\section{Patient and public involvement}

Before starting recruitment, randomly selected $\mathrm{CeD}$ and IBD patients reviewed the questionnaires and the information sheet designed to share details of the study for participants to facilitate better understanding.

\section{Blinding}

Blinding of personnel included in the study is presented in table 4 .

\section{Data management}

A subject identification number will be provided consecutively to every patient after inclusion. Subject identification numbers with sensitive data on patients (including the name, insurance number and date of enrolment) will be stored in a locked file separately from other data. De-identified data will be added to the source documentation stored in locked cabinets. Source documentation will be entered in an electronic case report file (e-CRF). The principal investigators will ensure that the data in an e-CRF are accurate, complete and legible (range checks for data values). E-CRFs will be stored on a secured server at the Institute for Translational Medicine, University of Pécs Medical School. Access to data will be restricted through a password system to personnel involved in data management. A three-level data check will be continuously performed, and final data will be finally approved by the principal investigator to ensure data quality.

To ensure precise data collection, administrative and medical staff members will be invited to participate in training sessions to familiarise them with the study requirements, standardised data recording and biological specimen collection.

The de-identified dataset will be delivered for the purpose of sharing on request.

\section{Statistical analysis}

First, descriptive statistics will entail a graphical presentation of data. Continuous variables will be reported as a central tendency with a measure of dispersion, while categorical variables will be reported as absolute and relative frequencies. Then, data will be analysed with Student's tests, methods of Variance Analysis, and regression models if data are normally distributed; otherwise, non-parametric tests will be introduced. $\chi^{2}$ or Fisher's tests will be used to analyse categorical variables. Multivariate analysis will be used to take the most important thrombotic factors into account (eg, the use of oral contraceptives and immunosuppressants, previous thrombotic history, smoking, comorbidities). A probability of less than 0.05 indicates a statistically significant difference between groups.

Only patients with a full dataset in their haemorheological and haemostatic profile will be included in the analysis. The following comparisons will be done: $\mathrm{CeD}$ versus control, tTG+CeD versus tTG- $\mathrm{CeD}, \mathrm{CeD}$ with good dietary adherence versus $\mathrm{CeD}$ with poor dietary adherence, IBD versus control, active IBD versus remitting IBD and Crohn's disease versus ulcerative colitis.

An interim analysis is planned after recruiting the target number of the first phase to calculate power. Audits are not necessary due to the case-control design.

\section{Biobank and accessory research}

After laboratory analysis, urine and blood (whole blood and plasma, at least $1 \mathrm{~mL}$ each) residues will be stored in the Institute for Translational Medicine Biobank at $-80^{\circ} \mathrm{C}$ for future studies (for at least 5 years). Additional samples will not be taken for storage purposes. Containers will be labelled with the subject identification number, and samples will be completely de-identified.

CeD patients will be offered an opportunity to participate in the 'Monitoring the prevalence, symptoms, complications and family history of $\mathrm{CeD}$ and the effect of a gluten-free diet-Coeliac registry' research 
project (approved by the Scientific and Research Ethics Committee of the Medical Research Council, Ref. No. 45098-2/2016/EKU).

\section{Protocol amendments and disseminating policy}

This protocol is the first version completed on 30 May 2018. If required, the online version will be updated in the ISRCTN registry. Major modifications should be permitted by the Regional and Local Research Ethics Committee.

The trial status is ongoing; recruitment began on 1 May 2018. The expected date of completion is 31 May 2019.

\section{DISCUSSION}

Recent guidelines on CeD do not make any recommendations on how to prevent and manage thrombotic events in CeD patients. ${ }^{3}{ }^{47}$ Gluten-free diet, which is the only approved treatment of the disease, may reduce or eliminate some thrombotic risk factors (eg, consequences of malabsorption and chronic inflammation) but it is uncertain whether the thrombotic risk completely normalises. ${ }^{57}$ With respect to malabsorption, intestinal mucosa does not recover in a high fraction of patients despite a long-term strict diet, particularly in those diagnosed in the adulthood. ${ }^{58}$ Whether CeD patients after a thrombotic event would benefit from a lifelong anticoagulation therapy has remained unclear. The need for thromboprophylaxis under prothrombotic circumstances, such as hospitalisation, pregnancy, or immobilisation should be further investigated.

IBD guidelines recommend that thromboprophylaxis should be considered in all inpatients and outpatients with an active disease. ${ }^{5960}$ In addition to disease severity, the choice of treatment influences the thrombotic risk as well. ${ }^{17}$ A tool of personalised thrombotic risk stratification including objective laboratory markers is awaited.

Our results can contribute to expanding our knowledge on the prothrombotic pathophysiological alteration in $\mathrm{CeD}$ and IBD, thereby providing the basis for future research.

\section{ETHICS AND DISSEMINATION}

Publication in a high-impact peer-reviewed journal is planned. We will adhere to authorship criteria for manuscripts submitted for publication set by the International Committee of Medical Journal Editors.

\footnotetext{
Author affiliations

1János Szentágothai Research Center, University of Pécs, Pécs, Hungary

${ }^{2}$ Clinical Medicine Doctoral School, University of Szeged, Szeged, Hungary

${ }^{3}$ Institute for Translational Medicine, Medical School, University of Pécs, Pécs,

Hungary

${ }^{4}$ Division of Cardiology and Angiology, First Department of Medicine, University of Pécs, Pécs, Hungary

${ }^{5}$ Division of Gastroenterology, First Department of Medicine, Medical School, University of Pécs, Pécs, Hungary

${ }^{6}$ Division of Hematology, First Department of Medicine, Medical School, University of Pécs, Pécs, Hungary
}

${ }^{7}$ Department of Biochemistry and Medical Chemistry, Medical School, University of Pécs, Pécs, Hungary

${ }^{8}$ Institute of Bioanalysis, Medical School, University of Pécs, Pécs, Hungary ${ }^{9}$ Department of Laboratory Medicine, Medical School, University of Pécs, Pécs, Hungary

${ }^{10}$ Department of Immunology and Biotechnology, Medical School, University of Pécs, Pécs, Hungary

Contributors JB is the Principal Investigator. ZS is the Trial Co-ordinator. ZS, $\mathrm{PH}$, $J B, A ́ V$ and $K T$ conceptualised the study, drafted and revised this manuscript. NF and EB planned and drafted the statistical analysis. PS, JB, BE and ÁV provided us with special expertise in the management of coeliac disease and inflammatory bowel patients. BC and PK are performing the haemorheological measurements and interpreting the results. AH, ÁN, TB and MT-F provided us with special expertise in hemostatic and immunological measurements. BK is contributing significantly to the biochemical analyses. IV planned and is carrying out the dietary assessment of the coeliac patients. KM, AS, ZS and PH are responsible for data management, administrative co-ordination and biological sampling; they drafted and revised the manuscript. All the authors have read and approved the final manuscript.

Funding The project is non-industry-funded. Study and centre costs are covered by the University of Pécs Medical School, by a Momentum Grant from the Hungarian Academy of Sciences (LP2014-10/2014), by a Highly Cited Publication Grant (KH 125678) from the National Research Development and Innovation Office, by GINOP 2.3.2-15-2016-00048 Stay Alive, EFOP-3.6.2-16-2017-0006, and EFOP-3.6.3VEKOP-16-2017-00009; and by the Translational Medicine Foundation. In addition, this project is supported by the ÚNKP-17-3-II and ÚNKP-18-3-I New National Excellence Programme, Ministry of Human Capacities. Funders have no influence on preparations, course, interpretation, or publication of results.

Competing interests None declared.

Patient consent for publication Not required.

Ethics approval The study was approved by the Regional and Local Research Ethics Committee, University of Pécs (Ref No 6917).

Provenance and peer review Not commissioned; externally peer reviewed.

Open access This is an open access article distributed in accordance with the Creative Commons Attribution Non Commercial (CC BY-NC 4.0) license, which permits others to distribute, remix, adapt, build upon this work non-commercially, and license their derivative works on different terms, provided the original work is properly cited, appropriate credit is given, any changes made indicated, and the use is non-commercial. See: http://creativecommons.org/licenses/by-nc/4.0/.

\section{REFERENCES}

1. El-Gabalawy H, Guenther LC, Bernstein CN. Epidemiology of immune-mediated inflammatory diseases: incidence, prevalence, natural history, and comorbidities. J Rheumatol Suppl 2010;85:2-10.

2. Pascual V, Dieli-Crimi R, López-Palacios N, et al. Inflammatory bowel disease and celiac disease: overlaps and differences. World $\mathrm{J}$ Gastroenterol 2014;20:4846-56.

3. Bai JC, Ciacci C. World gastroenterology organisation global guidelines: Celiac disease February 2017. J Clin Gastroenterol 2017:51:755-68.

4. Ludvigsson JF, Leffler DA, Bai JC, et al. The Oslo definitions for coeliac disease and related terms. Gut 2013;62:43-52.

5. Kurppa K, Paavola A, Collin P, et al. Benefits of a gluten-free diet for asymptomatic patients with serologic markers of celiac disease. Gastroenterology 2014;147:610-7.

6. Zhang YZ, Li YY, Yy L. Inflammatory bowel disease: pathogenesis. World J Gastroenterol 2014;20:91-9.

7. Molodecky NA, Soon IS, Rabi DM, et al. Increasing incidence and prevalence of the inflammatory bowel diseases with time, based on systematic review. Gastroenterology 2012;142:46-54.

8. Kaplan GG. The global burden of IBD: from 2015 to 2025. Nat Rev Gastroenterol Hepatol 2015;12:720-7.

9. Ernst E, Hein A, Meurer M, et al. Blood rheology in lupus erythematosus. Ann Rheum Dis 1991;50:710-2.

10. Shurkhina ES, Nesterenko VM, Lisovskaya IL, et al. Detection of stages of autoimmune hemolytic anemia by evaluating erythrocyte deformability and density. Bull Exp Biol Med 2004;138:280-3.

11. Spengler MI, Svetaz MJ, Leroux MB, et al. Erythrocyte aggregation in patients with systemic lupus erythematosus. Clin Hemorheol Microcirc 2011;47:279-85. 
12. Adams MJ, Palatinus AA, Harvey AM, et al. Impaired control of the tissue factor pathway of blood coagulation in systemic lupus erythematosus. Lupus 2011;20:1474-83.

13. Costallat LT, Ribeiro CC, Annichino-Bizzacchi JM. Antithrombin, protein $\mathrm{S}$ and protein $\mathrm{C}$ and antiphospholipid antibodies in systemic lupus erythematosus. Sangre 1998;43:345-8.

14. Kordich LC, Forastiero RR, Basilotta E, et al. Natural inhibitors of blood coagulation and fibrinolysis in patients with lupus anticoagulant. Blood Coagul Fibrinolysis 1992;3:765-72.

15. Zöller B, Li X, Sundquist J, et al. Risk of pulmonary embolism in patients with autoimmune disorders: a nationwide follow-up study from Sweden. Lancet 2012;379:244-9.

16. Ungprasert $P$, Wijarnpreecha $K$, Tanratana P. Risk of venous thromboembolism in patients with celiac disease: A systematic review and meta-analysis. J Gastroenterol Hepatol 2016;31:1240-5.

17. Sarlos P, Szemes K, Hegyi P, et al. Steroid but not biological therapy elevates the risk of venous thromboembolic events in inflammatory bowel disease: a meta-analysis. J Crohns Colitis 2018;12:489-98.

18. Chang HH, Chiang BL. The diagnosis and classification of autoimmune coagulopathy: an updated review. Autoimmun Rev 2014;13:587-90.

19. Park KH, Kim U, Choi KU, et al. Hemorheologic alterations in patients with type 2 diabetes mellitus presented with an acute myocardial infarction. Diabetes Metab J 2018;42:155-63.

20. Vayá A, Rivera L, de la Espriella R, et al. Red blood cell distribution width and erythrocyte deformability in patients with acute myocardia infarction. Clin Hemorheol Microcirc 2015;59:107-14.

21. Zorio E, Murado J, Arizo D, et al. Haemorheological parameters in young patients with acute myocardial infarction. Clin Hemorheol Microcirc 2008;39:33-41.

22. Banerjee R, Nageswari K, Puniyani RR. Association of hemorheological parameters and risk of stroke in hypertensives of Indian origin. Clin Exp Hypertens 2000;22:687-94.

23. Wolberg AS, Aleman MM, Leiderman K, et al. Procoagulant activity in hemostasis and thrombosis: Virchow's triad revisited. Anesth Analg 2012;114:275-85.

24. Lerner A, Blank M. Hypercoagulability in celiac disease-an update. Autoimmun Rev 2014;13:1138-41.

25. Dumic I, Martin S, Salfiti N, et al. Deep venous thrombosis and bilateral pulmonary embolism revealing silent celiac disease: Case report and review of the literature. Case Rep Gastrointest Med 2017;2017:1-8

26. Berthoux E, Fabien N, Chayvialle JA, et al. [Adult celiac disease with thrombosis: a case series of seven patients. Role of thrombophilic factors]. Rev Med Interne 2011;32:600-4.

27. Lerner A, Agmon-Levin N, Shapira Y, et al. The thrombophilic network of autoantibodies in celiac disease. BMC Med 2013;11:89.

28. Hallert C, Grant C, Grehn S, et al. Evidence of poor vitamin status in coeliac patients on a gluten-free diet for 10 years. Aliment Pharmacol Ther 2002;16:1333-9.

29. Shamir R, Shoenfeld Y, Blank M, et al. The prevalence of coeliac disease antibodies in patients with the antiphospholipid syndrome. Lupus 2003;12:394-9.

30. Johannesdottir SA, Erichsen R, Horváth-Puhó E, et al. Coeliac disease and risk of venous thromboembolism: a nationwide population-based case-control study. Br J Haematol 2012;157:499-501.

31. Ciaccio EJ, Lewis SK, Biviano AB, et al. Cardiovascular involvement in celiac disease. World J Cardiol 2017;9:652-66.

32. Verma AK, Gatti S, Galeazzi T, et al. Gluten contamination in naturally or labeled gluten-free products marketed in Italy. Nutrients 2017;9:115

33. Mitu O, Alexandrescu DM, Cătălina $\mathrm{M}$, et al. Is there a cardiovascular risk in inflammatory bowel diseases? Rev Med Chir Soc Med Nat lasi 2014;118:918-23.

34. Kristensen SL, Ahlehoff O, Lindhardsen J, et al. Disease activity in inflammatory bowel disease is associated with increased risk of myocardial infarction, stroke and cardiovascular death-a Danish nationwide cohort study. PLoS One 2013;8:e56944.

35. Cakal B, Gokmen A, Yalinkilic M, et al. Natural anticoagulant protein levels in Turkish patients with inflammatory bowel disease. Blood Coagul Fibrinolysis 2010;21:118-21.

36. Heneghan MA, Cleary B, Murray M, et al. Activated protein C resistance, thrombophilia, and inflammatory bowel disease. Dig Dis Sci 1998:43:2164-7.

37. Kohoutova D, Pecka M, Cihak M, et al. Prevalence of hypercoagulable disorders in inflammatory bowel disease. Scand $J$ Gastroenterol 2014;49:287-94.
38. Yurekli BP, Aksoy DY, Aybar M, et al. The search for a common thrombophilic state during the active state of inflammatory bowe disease. J Clin Gastroenterol 2006;40:809-13.

39. Akman $\mathrm{T}$, Akarsu M, Akpinar $\mathrm{H}$, et al. Erythrocyte deformability and oxidative stress in inflammatory bowel disease. Dig Dis Sci 2012:57:458-64.

40. Novacek G, Vogelsang H, Genser D, et al. Changes in blood rheology caused by Crohn's disease. Eur J Gastroenterol Hepatol 1996:8:1089-93.

41. Zilberman L, Rogowski O, Rozenblat M, et al. Inflammation-related erythrocyte aggregation in patients with inflammatory bowel disease. Dig Dis Sci 2005;50:677-83.

42. Lobo AJ, Jones SC, Juby LD, et al. Plasma viscosity in inflammatory bowel disease. J Clin Pathol 1992;45:54-7.

43. Danese S, Papa A, Saibeni S, et al. Inflammation and coagulation in inflammatory bowel disease: The clot thickens. Am J Gastroenterol 2007;102:174-86.

44. Magro F, Soares JB, Fernandes D. Venous thrombosis and prothrombotic factors in inflammatory bowel disease. World $J$ Gastroenterol 2014;20:4857-72.

45. Chan AW, Tetzlaff JM, Altman DG, et al. SPIRIT 2013 statement: defining standard protocol items for clinical trials. Ann Intern Med 2013;158:200-7.

46. Márta K, Szabó AN, Pécsi D, et al. High versus low energy administration in the early phase of acute pancreatitis (GOULASH trial): protocol of a multicentre randomised double-blind clinical trial. BMJ Open 2017;7:e015874.

47. Husby S, Koletzko S, Korponay-Szabó IR, et al. European Society for Pediatric Gastroenterology, Hepatology, and Nutrition guidelines for the diagnosis of coeliac disease. J Pediatr Gastroenterol Nutr 2012:54:136-60.

48. Rubio-Tapia A, Hill ID, Kelly CP, et al. ACG clinical guidelines: diagnosis and management of celiac disease. Am J Gastroenterol 2013;108:656-76.

49. Dignass A, Eliakim R, Magro F, et al. Second European evidencebased consensus on the diagnosis and management of ulcerative colitis part 1: definitions and diagnosis. $J$ Crohns Colitis 2012;6:965-90.

50. Dignass A, Van Assche G, Lindsay JO, et al. The second European evidence-based Consensus on the diagnosis and management of Crohn's disease: Current management. J Crohns Colitis 2010;4:28-62.

51. Svedlund J, Sjödin I, Dotevall G. GSRS--a clinical rating scale for gastrointestinal symptoms in patients with irritable bowel syndrome and peptic ulcer disease. Dig Dis Sci 1988;33:129-34.

52. Schroeder KW, Tremaine WJ, Ilstrup DM. Coated oral 5 -aminosalicylic acid therapy for mildly to moderately active ulcerative colitis. A randomized study. N Engl J Med 1987;317:1625-9.

53. Modigliani R, Mary JY, Simon JF, et al. Clinical, biological, and endoscopic picture of attacks of Crohn's disease. Evolution on prednisolone. Groupe d'Etude Thérapeutique des Affections Inflammatoires Digestives. Gastroenterology 1990;98:811-8.

54. Silvester JA, Weiten D, Graff LA, et al. Living gluten-free: adherence, knowledge, lifestyle adaptations and feelings towards a gluten-free diet. J Hum Nutr Diet 2016;29:374-82.

55. Hindryckx P, Levesque BG, Holvoet T, et al. Disease activity indices in coeliac disease: systematic review and recommendations for clinical trials. Gut 2018;67:61-9.

56. Baskurt OK, Boynard M, Cokelet GC, et al. New guidelines for hemorheological laboratory techniques. Clin Hemorheol Microcirc 2009;42:75-97.

57. Beyrouti R, Mansour M, Kacem A, et al. Recurrent cerebral venous thrombosis revealing celiac disease: an exceptional case report. Acta Neurol Belg 2017;117:341-3.

58. Szakács Z, Mátrai P, Hegyi P, et al. Younger age at diagnosis predisposes to mucosal recovery in celiac disease on a gluten-free diet: A meta-analysis. PLoS One 2017;12:e0187526.

59. Gionchetti P, Dignass A, Danese S, et al. 3rd European evidencebased consensus on the diagnosis and management of crohn's disease 2016: Part 2: Surgical management and special situations. $J$ Crohns Colitis 2017;11:135-49.

60. Nguyen GC, Bernstein CN, Bitton A, et al. Consensus statements on the risk, prevention, and treatment of venous thromboembolism in inflammatory bowel disease: Canadian Association of Gastroenterology. Gastroenterology 2014;146:835-48. 